May 2007

\title{
Gravitino in six-dimensional warped supergravity
}

\author{
Hyun Min Lee ${ }^{a, *}$ and Antonios Papazoglou ${ }^{b, c, * *}$ \\ ${ }^{a}$ Department of Physics, Carnegie Mellon University, \\ 5000 Forbes Avenue, Pittsburgh, PA 15213, USA. \\ ${ }^{b} \mathrm{APC}^{1}, 10$ rue Alice Domon et Léonie Duquet, \\ 75205 Paris Cedex 13, France. \\ ${ }^{c}$ GReCO/IAP 2 , 98 bis Boulevard Arago, \\ 75014 Paris, France.
}

\begin{abstract}
We consider the gravitino spectrum for the general warped solution in a specific six-dimensional gauged supergravity. We find that although the brane tensions introduced at the conical singularities break the bulk supersymmetry explicitly, massless modes of the gravitino can exist with a nontrivial wave function profile, due to a nonzero $U(1)_{R}$ gauge flux. We also compute the wave function and the mass spectrum of Kaluza-Klein massive modes of the gravitino explicitly. We show that the introduction of a gravitino mass term on a regularized brane can give a suppressed effective gravitino mass compared to the compactification scale, due to the delocalization of the wave function of the zero-mode gravitino.
\end{abstract}

PACS codes: 04.50.+h, 11.25.Mj, 04.65.+e.

Keywords: Supergravity, Extra dimensions, Conical singularity, Supersymmetry breaking.

* e-mail address: hmlee@andrew.cmu.edu

** e-mail address: papazogl@iap.fr

\footnotetext{
${ }^{1}$ UMR 7164(CNRS, Université Paris 7, CEA, Obervatoire de Paris)

${ }^{2}$ UMR 7095(CNRS, Université Paris 6)
} 


\section{Introduction}

There has been a lot of interest in brane world models in higher dimensions with the hope to solve the particle physics problems and give a hint for physics beyond the Standard Model (SM) in a different context. Particularly, in order to ameliorate the hierarchy problem of the Higgs mass, models with extra dimensions compactified on a large flat [1] or small warped [2] space were suggested as an alternative to the weak-scale supersymmetry (SUSY). Furthermore, regarding the cosmological constant problem which has been one of the most notorious problems as dictated by a no-go theorem [3], the self-tuning mechanism in higher dimensions [4] was suggested. This may give a better understanding of the cosmological constant problem, although one has only the SM quantum corrections confined on a brane under control. In particular, brane world models in six dimensions have drawn much attention because the brane tension generates a nonzero deficit angle in extra dimensions without curving the 4D spacetime [5]. This feature has been first pursued in the framework of spontaneous compactification due to gauge fluxes in 6D Einstein-Maxwell theory [6], but ended up with a fine-tuning condition for the brane tension due to flux quantization or conservation [7]. Furthermore, we still need some symmetry to ensure that a bulk tuning condition is stable against the quantum corrections.

The Salam-Sezgin (SS) supergravity [8] has drawn a renewed interest due to the possibility of attacking both brane and bulk fine-tuning problems encountered in the nonsupersymmetric models. In this model, Salam and Sezgin obtained a spontaneous compactification on a sphere with $U(1)_{R}$ flux to get the 4 D Minkowski spacetime and showed that $4 \mathrm{D} \mathcal{N}=1$ SUSY survives or there is a massless chiral gravitino in four dimensions. The most general warped non-singular (no worse than conical) solutions with 4D maximal symmetry have been recently found to be a warped product of the 4D Minkowski space and a two dimensional compact manifold (the "football-shaped" space [9], the general warped solution with axial symmetry [10] or the general warped solution without axial symmetry [11]). Nonetheless, there is still a fine-tuning between brane tensions due to the flux quantization. It has been shown, on the other hand, that there are warped singular solutions with 4D curved spacetime [12]. The stability analysis of the warped background has been done for scalar perturbations [13] and bulk gauge fields and fermions [14].

The warping requires the existence of conical singularities on which codimension-two branes are located, so $\mathcal{N}=1$ SUSY is broken explicitly by nonzero brane tensions ${ }^{3}$. Nevertheless, it has been suggested that the SUSY breakdown at the branes might be suppressed not to give a large quantum correction in the bulk [16]. The Casimir effect has been discussed for flux compactifications in non-SUSY and SUSY models [17]. We will, thus, discuss the model at the classical level, not taking into account the transmission of the SUSY breaking from the brane sources to the bulk. In this paper, we study the gravitino equation for the general warped solution in an anomaly free model with the gauge group $E_{6} \times E_{7} \times U(1)_{R}$ in six-dimensional gauged supergravity [18] ${ }^{4}$. The difference from

\footnotetext{
${ }^{3}$ Such explicit SUSY breaking localized terms are a usual addition in supergravity models, e.g., $\overline{D 3}$ branes in throat geometries [15].

${ }^{4}$ For recent studies of six-dimensional supergravities, see Ref. [19].
} 
the SS model is that on top of the $U(1)_{R}$ flux, we can also turn on the abelian flux of the non-abelian gauge fields, for instance, $E_{6}$, but the form of the general warped solution is maintained. In this case, both SUSY and the GUT group can be broken upon the compactification so the model could provide an alternative to orbifold models. It has been shown that $E_{6}$ is broken down to $S O(10)$ and the adjoint fermions of $E_{6}$ survive as two chiral 16's of $S O(10)$ [18].

By solving the gravitino equation, we find that there exist massless modes of the gravitino for a nonzero $U(1)_{R}$ flux, even with nonzero brane tensions and/or abelian flux of $E_{6}$. We show that the wave function of the massless modes of the gravitino have a nontrivial profile in the extra dimensions. Moreover, we also obtain the wave function and the mass spectrum for the massive modes of the gravitino. Although we can show from the bulk SUSY transformation that SUSY is broken for the warped solution, the appearance of massless modes of the gravitino tells us that the gravitino mass must be determined by interaction terms beyond the bulk action and the brane tensions.

However, we cannot consider brane matter terms on the codimension-2 branes without introducing singularities [20]. It is imperative that the brane is regularized by either acquiring some thickness [21], or by the reduction of its codimension [22,23]. Considering the latter method (codimension reduction) and a brane-induced gravitino mass term on the regularized brane, we found that the gravitino mass can be suppressed compared to the compactification scale due to the delocalization of the zero-mode gravitino.

The paper is organized as follows. We first review the warped solution in 6D gauged supergravity. Then, we consider the SUSY transformation and the gravitino action in the warped background. Further, we present the wave functions of the massless modes of the gravitino and the mass spectrum of KK massive modes of the gravitino. Next, we introduce brane-induced gravitino mass terms and estimate the effective gravitino mass related to the value of the wave function of the zero-mode gravitino on the brane. Finally, the conclusions are drawn.

\section{The model}

We consider an anomaly-free model [18] in 6D gauged supergravity where the bulk gauge group is $E_{6} \times E_{7} \times U(1)_{R}$ with gauge couplings $g_{6}, g_{7}$ and $g_{1}$. By setting the Kalb-Ramond field and the hyperscalars to zero, the bosonic part of the bulk action ${ }^{5}$ is given by

$$
e^{-1} \mathcal{L}_{b}=R-\frac{1}{4}\left(\partial_{M} \phi\right)^{2}-8 g_{1}^{2} e^{-\frac{1}{2} \phi}-\frac{1}{4} e^{\frac{1}{2} \phi}\left(F_{M N}^{2}+F_{6 M N}^{I 2}+F_{7 M N}^{I^{\prime} 2}\right) .
$$

When all non-abelian gauge fields vanish, the above action becomes the one of the SalamSezgin model [8]. The case with only the $U(1) \subset E_{6}$ being nonzero was considered for a GUT breaking in [18]. In the present paper, we take the more general case where both

\footnotetext{
${ }^{5}$ For comparison with the Salam-Sezgin [8] notation, we note that $g_{S S}=2 g_{1}, A_{M}^{S S}=A_{M} / 2, \sigma=\phi / 2$, and $\mathcal{L}_{S S}=\mathcal{L} / 4$. We set the $6 \mathrm{D}$ fundamental scale to $\kappa=1$.
} 
$U(1)_{R}$ and $U(1)$ are nonzero. In this case, it has been known that supersymmetry is broken completely and $E_{6}$ is reduced to $S O(10)$ [18].

Assuming axial symmetry in the internal space, the general warped solution with $U(1)_{R}$, $U(1)$ fluxes can be found to give rise to four-dimensional Minkowski vacua (i.e., with zero effective cosmological constant) with the following form [10]

$$
\begin{aligned}
d s^{2} & =W^{2}(r) \eta_{\mu \nu} d x^{\mu} d x^{\nu}+R^{2}(r)\left(d r^{2}+\lambda^{2} \Theta^{2}(r) d \theta^{2}\right) \\
F_{1 r \theta} & =\lambda q \frac{\Theta R^{2}}{W^{6}} \cos \alpha, \quad T_{I} F_{6 r \theta}^{I}=T_{0} \lambda q \frac{\Theta R^{2}}{W^{6}} \sin \alpha \\
\phi & =4 \ln W
\end{aligned}
$$

with

$$
\begin{array}{r}
R=\frac{W}{f_{0}}, \quad \Theta=\frac{r}{W^{4}}, \\
W^{4}=\frac{f_{1}}{f_{0}}, \quad f_{0}=1+\frac{r^{2}}{r_{0}^{2}}, \quad f_{1}=1+\frac{r^{2}}{r_{1}^{2}},
\end{array}
$$

where $q$ is a constant denoting the magnetic flux, $T_{0}$ is the $U(1)$ generator of $E_{6}$ and the two radii are given by

$$
r_{0}^{2}=\frac{1}{2 g_{1}^{2}}, \quad r_{1}^{2}=\frac{8}{q^{2}} .
$$

The angle $\alpha$ denotes the distribution of the fluxes in the $U(1)_{R}$ and the $U(1)$ directions. From eq. (3), we get the nonzero component of gauge fields in the patch including $r=0$ as

$$
g_{1} A_{1 \theta}=-n_{1}\left(\frac{1}{f_{1}}-1\right), \quad g_{6} T_{I} A_{6 \theta}^{I}=-T_{0} n_{6}\left(\frac{1}{f_{1}}-1\right) .
$$

where we have taken into account the quantization conditions of the gauge fluxes

$$
\frac{4 \lambda g_{1} \cos \alpha}{q}=n_{1}, \quad \frac{4 \lambda g_{6} \sin \alpha}{q}=n_{6}, \quad n_{1}, n_{6}=\text { integer } .
$$

In this general solution, the metric has two conical singularities, one at $r=0$ and the other at $r=\infty$, with deficit angles $\delta_{s}$ (supported by brane tensions $V_{s}=2 \delta_{s}$ ) given by

$$
\begin{aligned}
& \frac{\delta_{0}}{2 \pi}=1-\lambda, \\
& \frac{\delta_{\infty}}{2 \pi}=1-\lambda \frac{r_{1}^{2}}{r_{0}^{2}}=1-\frac{1}{\lambda}\left(n_{1}^{2}+\frac{g_{1}^{2}}{g_{6}^{2}} n_{6}^{2}\right) .
\end{aligned}
$$

These brane terms, as it will be discussed later, are explicitly non-supersymmetric. Let us note also, that the gauge field Bianchi identities are satisfied as long as there is no coupling of the brane to the bulk gauge field [9].

For $r_{0}=r_{1}$, i.e. $q=4 g_{1}$, we get the unwarped solution with football shaped extra dimensions. In this case, we get $\lambda^{2}=n_{1}^{2}+n_{6}^{2} g_{1}^{2} / g_{6}^{2}$ and if both $n_{1}$ and $n_{6}$ are nonzero, the 
angle deficit has to be negative (and so has to be the brane tension). If additionally $\lambda=1$, the unwarped solution is possible with no branes present. The latter happens only for the following two cases: $\left(n_{1}, n_{6}\right)=(1,0)$ or $\left(n_{1}, n_{6}\right)=\left(0, g_{6} / g_{1}\right)$. So, in this case, only either of $U(1)$ fluxes can be nonzero.

Finally, by defining

$$
d \rho=R d r, \quad a=\lambda R \Theta,
$$

the metric can be expressed in a Gaussian normal coordinate system as

$$
d s^{2}=W^{2} \eta_{\mu \nu} d x^{\mu} d x^{\nu}+d \rho^{2}+a^{2} d \theta^{2}
$$

\section{Explicit supersymmetry breaking and gravitino dy- namics in the warped background}

In this section we will discuss the gravitino spectrum in the general warped background and the existence of gravitino zero modes even in some cases where supersymmetry is broken by the presence of the brane tension terms. In order to do this analysis, we need the spinor part of the action and in particular the part that is quadratic in fermionic terms. This is given by $[18]^{6}$

$$
\begin{aligned}
e^{-1} \mathcal{L}_{f}= & \bar{\psi}_{M} \Gamma^{M N P} \mathcal{D}_{N} \psi_{P}+\bar{\chi} \Gamma^{M} \mathcal{D}_{M} \chi+\bar{\lambda} \Gamma^{M} \mathcal{D}_{M} \lambda+\bar{\lambda}_{6} \Gamma^{M} \mathcal{D}_{M} \lambda_{6} \\
& +\frac{1}{4}\left(\partial_{M} \phi\right)\left(\bar{\psi}_{N} \Gamma^{M} \Gamma^{N} \chi+\text { h.c. }\right)+\sqrt{2} g_{1} e^{-\frac{1}{4} \phi}\left(i \bar{\psi}_{M} \Gamma^{M} \lambda_{1}-i \bar{\chi} \lambda_{1}+\text { h.c. }\right) \\
& -\frac{1}{4 \sqrt{2}} e^{\frac{1}{4} \phi}\left\{F_{1 M N}\left(\bar{\psi}_{Q} \Gamma^{M N} \Gamma^{Q} \lambda_{1}+\bar{\chi} \Gamma^{M N} \lambda_{1}\right)\right. \\
& \left.+F_{6 M N}^{I}\left(\bar{\psi}_{Q} \Gamma^{M N} \Gamma^{Q} \lambda_{6}^{I}+\bar{\chi} \Gamma^{M N} \lambda_{6}^{I}\right)+\text { h.c. }\right\},
\end{aligned}
$$

where the covariant derivative for any fermion $\psi$ is defined as

$$
\mathcal{D}_{M} \psi=\left(\partial_{M}+\frac{1}{4} \omega_{M A B} \Gamma^{A B}-i g_{1} A_{M}\right) \psi
$$

The above spinors are chiral with handednesses

$$
\Gamma^{7} \psi_{M}=+\psi_{M}, \quad \Gamma^{7} \chi=-\chi, \quad \Gamma^{7} \lambda_{1}=+\lambda_{1}, \quad \Gamma^{7} \lambda_{6}=+\lambda_{6} .
$$

Taking into account that $\Gamma^{7}=\sigma^{3} \otimes \mathbf{1}$ (see Appendix A), the 6D (8-component) spinors can be decomposed to $6 \mathrm{D}$ Weyl (4-component) spinors as

$$
\psi_{M}=\left(\tilde{\psi}_{M}, 0\right)^{T}, \quad \chi=(0, \tilde{\chi})^{T}, \quad \lambda_{1}=\left(\tilde{\lambda}_{1}, 0\right)^{T}, \quad \lambda_{6}=\left(\tilde{\lambda}_{6}, 0\right)^{T}
$$

\footnotetext{
${ }^{6}$ In comparison with the SS notation [8], all fermions are rescaled as $\psi_{S S}=\psi / 2$.
} 


\subsection{Supersymmetry transformations}

Before presenting the gravitino spectrum, let us discuss the supersymmetry of the vacua in question. For the general background with fluxes, the nontrivial bulk supersymmetry transformations of fermions are the ones for dilatino $\chi$, gravitino $\psi_{M}, U(1)_{R}$ gaugino $\lambda_{1}$ and $E_{6}$ gauginos $\lambda_{6}^{a}[18]$ :

$$
\begin{aligned}
\delta \chi & =-\frac{1}{4}\left(\partial_{m} \phi\right) \Gamma^{m} \varepsilon \\
\delta \lambda_{1} & =\frac{1}{4 \sqrt{2}} e^{\frac{1}{4} \phi} F_{1 m n} \Gamma^{m n} \varepsilon-i \sqrt{2} g_{1} e^{-\frac{1}{4} \phi} \varepsilon, \\
\delta\left(T_{I} \lambda_{6}^{I}\right) & =\frac{1}{4 \sqrt{2}} e^{\frac{1}{4} \phi}\left(T_{I} F_{6 m n}^{I}\right) \Gamma^{m n} \varepsilon, \\
\delta \psi_{M} & =\mathcal{D}_{M} \varepsilon
\end{aligned}
$$

where the spinor parameter $\varepsilon$ is chiral, with $\Gamma^{7} \varepsilon=+\varepsilon$, so that $\varepsilon=(\tilde{\varepsilon}, 0)^{T}$.

Using the solution that we presented in the previous section, the above transformations give

$$
\begin{aligned}
\delta \chi & =-\frac{W^{\prime}}{W}\left[\cos \theta \sigma^{1} \otimes \gamma^{5}+\sin \theta \sigma^{2} \otimes \mathbf{1}\right] \varepsilon \\
\delta \lambda_{1} & =\frac{i}{\sqrt{2} W} 2 g_{1} \sigma^{3} \otimes\left[\frac{q}{4 g_{1}} \frac{1}{W^{4}} \cos \alpha \gamma^{5}-1\right] \varepsilon \\
\delta\left(T_{I} \lambda_{6}^{I}\right) & =T_{0} i \sqrt{2} \frac{q}{4} \frac{1}{W^{4}} \sin \alpha \sigma^{3} \otimes \gamma^{5} \varepsilon \\
\delta \psi_{\mu} & =\left[\partial_{\mu}+\frac{1}{2} W^{\prime} \sigma^{3} \otimes \gamma_{\mu}\left(\cos \theta \gamma^{5}+i \sin \theta\right)\right] \varepsilon \\
\delta \psi_{\rho} & =\partial_{\rho} \varepsilon, \\
\delta \psi_{\theta} & =\left[\partial_{\theta}+\frac{i}{2}\left(1+\frac{\lambda}{W^{4}}\left(1-\frac{2}{f_{0}}\right)+3 \lambda r \frac{W^{\prime}}{W^{5}}\right) \sigma^{3} \otimes \gamma^{5}+i \lambda \frac{4 g_{1}}{q} \cos \alpha\left(\frac{1}{f_{1}}-1\right)\right] \varepsilon .
\end{aligned}
$$

In particular, for the sphere solution with only $U(1)_{R}$ flux turned on, i.e. the SalamSezgin vacuum with $\lambda=1,\left(n_{1}, n_{6}\right)=(1,0), q=4 g_{1}$, half of the supersymmetries are preserved [8]. In this case, the SUSY conditions for constant spinors are

$$
\begin{aligned}
\delta \chi & =\delta\left(T_{I} \lambda_{6}^{I}\right)=\delta \psi_{\rho}=\delta \psi_{\mu}=0 \\
\delta \lambda_{1} & =\frac{1}{2 \sqrt{2}} i q \sigma^{3} \otimes\left(\gamma_{5}-1\right) \varepsilon=0 \\
\delta \psi_{\theta} & =\left[\partial_{\theta}-i\left(\frac{1}{f_{0}}-1\right) \sigma^{3} \otimes\left(\gamma_{5}-1\right)\right] \varepsilon=0 .
\end{aligned}
$$

Thus, it is obvious from the above that there exists a constant $4 \mathrm{D}$ Killing Weyl spinor $\tilde{\varepsilon}_{L}$, with $\tilde{\varepsilon}=\left(\tilde{\varepsilon}_{L}, 0\right)^{T}$, which preserves $4 \mathrm{D} \mathcal{N}=1$ supersymmetry. In this case, it was shown that there exists a chiral massless mode of gravitino [8] due to the cancellation between spin and $U(1)_{R}$ connections. 
On the other hand, the above SUSY transformations show that SUSY can be spontaneously broken by a nonzero flux along the $E_{6}$ even without conical singularities, i.e. $T_{I} F_{m n}^{I} \neq 0$. This is partially because there is no potential term in the variation to cancel the gauge field related part in (20). The fermion SUSY transformations for constant $\varepsilon$ then read

$$
\begin{aligned}
\delta \chi & =\delta \psi_{\rho}=\delta \psi_{\mu}=0 \\
\delta \lambda_{1} & =-i \sqrt{2} g_{1} \sigma^{3} \otimes \mathbf{1} \varepsilon \\
\delta\left(T_{I} \lambda_{6}^{I}\right) & =T_{0} i \sqrt{2} g_{1} \sigma^{3} \otimes \gamma^{5} \varepsilon \\
\delta \psi_{\theta} & =\left[\partial_{\theta}-i\left(\frac{1}{f_{0}}-1\right) \sigma^{3} \otimes \gamma^{5}\right] \varepsilon .
\end{aligned}
$$

Upon introducing conical singularities to the Salam-Sezgin solution, by deforming the space with non-SUSY brane tensions [10], it can be seen from (22)-(27) that the SUSY is broken completely in the bulk due to the absence of a globally well-defined Killing spinor for the conical geometries [24]. As will be seen in the next sections, however, the explicit SUSY breakdown does not mean necessarily the absence of a massless mode of the gravitino.

\subsection{The "4D gravitino" equation of motion}

For the background solution of the previous section, we can rearrange the fermionic part of the action (14) as

$$
\begin{aligned}
e^{-1} \mathcal{L}_{f}= & \bar{\chi} \Gamma^{M} \mathcal{D}_{M} \chi+\bar{\lambda} \Gamma^{M} \mathcal{D}_{M} \lambda+\bar{\lambda}_{6} \Gamma^{M} \mathcal{D}_{M} \lambda_{6}+\bar{\psi}_{m} \Gamma^{m \lambda n} \mathcal{D}_{\lambda} \psi_{n}+e^{-1} \mathcal{L}_{\text {mix }} \\
& +\bar{\psi}_{\mu} \Gamma^{\mu \nu \lambda} \mathcal{D}_{\nu} \psi_{\lambda}+\bar{\psi}_{\mu} \Gamma^{\mu m \lambda} \mathcal{D}_{m} \psi_{\lambda} \\
& +\left(-\bar{\psi}_{\mu} g^{\mu \lambda} \Gamma^{m} \mathcal{D}_{\lambda} \psi_{m}+\bar{\psi}_{\mu} \Gamma^{\mu} \eta+\text { h.c. }\right),
\end{aligned}
$$

where $\mathcal{L}_{\text {mix }}$ contains the mixing terms between spin- $\frac{1}{2}$ components. The linear combination of the spin- $\frac{1}{2}$ fermions

$$
\begin{aligned}
\eta= & \Gamma^{\lambda} \Gamma^{m} \mathcal{D}_{\lambda} \psi_{m}+\Gamma^{m n} \mathcal{D}_{m} \psi_{n}-\frac{1}{4}\left(\partial_{m} \phi\right) \Gamma^{m} \chi+i \sqrt{2} g_{1} e^{-\frac{1}{4} \phi} \lambda_{1} \\
& -\frac{1}{4 \sqrt{2}} e^{\frac{1}{4} \phi}\left(F_{1 m n} \Gamma^{m n} \lambda_{1}+F_{6 m n}^{I} \Gamma^{m n} \lambda_{6}^{I}\right),
\end{aligned}
$$

plays the role of the would-be Goldstone fermion and mixes with the "4D gravitino" $7 \psi_{\mu}$. Even if supersymmetry is broken explicitly by brane sources, we can get rid of the mixing terms with the spin- $\frac{1}{2}$ fermions by the redefinition of the $4 \mathrm{D}$ gravitino. Therefore, in order to get the mass spectrum of the "4D gravitino", we only have to consider the second line of the action (35)

$$
e^{-1} \mathcal{L}_{\psi}=\bar{\psi}_{\mu} \Gamma^{\mu \nu \lambda} \mathcal{D}_{\nu} \psi_{\lambda}+\bar{\psi}_{\mu} \Gamma^{\mu n \lambda} \mathcal{D}_{n} \psi_{\lambda}
$$

\footnotetext{
${ }^{7}$ We use the term "4D gravitino" to denote the $4 \mathrm{D}$ vector component of the gravitino before dimensional reduction.
} 
where

$$
\mathcal{D}_{\nu}=\partial_{\nu}+\frac{1}{2} \omega_{\nu \alpha 5} \Gamma^{\alpha 5}+\frac{1}{2} \omega_{\nu \alpha 6} \Gamma^{\alpha 6}, \quad \mathcal{D}_{n}=\partial_{n}+\frac{1}{2} \omega_{n 56} \Gamma^{56}-i g_{1} A_{1 n},
$$

and $\Gamma^{\mu n \lambda}=-\Gamma^{\mu \lambda} \Gamma^{n}$. From this action, we derive easily the equation of motion for the "4D gravitino" as

$$
\Gamma^{\mu \nu \lambda}\left(\partial_{\nu}+\frac{1}{2} \omega_{\nu \alpha 5} \Gamma^{\alpha 5}+\frac{1}{2} \omega_{\nu \alpha 6} \Gamma^{\alpha 6}\right) \psi_{\lambda}-\Gamma^{\mu \lambda} \Gamma^{n}\left(\partial_{n}+\frac{1}{2} \omega_{n 56} \Gamma^{56}-i g_{1} A_{1 n}\right) \psi_{\lambda}=0 .
$$

Using the vielbein and the spin connection obtained in the Appendix A, we can rewrite the gravitino equation as

$$
\begin{aligned}
0= & W^{-1} \sigma^{1} \otimes \gamma^{\alpha \beta \gamma} \delta_{\gamma}^{\mu}\left[\partial_{\beta}+\frac{1}{2} W^{\prime}\left(\sigma^{0} \otimes \gamma_{\beta} \gamma^{5} \cos \theta+i \sigma^{3} \otimes \gamma_{\beta} \sin \theta\right)\right] \psi_{\mu} \\
& -\sigma^{0} \otimes \gamma^{\alpha \gamma} \delta_{\gamma}^{\mu}\left(\sigma^{1} \otimes \gamma^{5} \cos \theta+\sigma^{2} \otimes \mathbf{1} \sin \theta\right) \partial_{\rho} \psi_{\mu} \\
& -\sigma^{0} \otimes \gamma^{\alpha \gamma} \delta_{\gamma}^{\mu}\left(-\sigma^{1} \otimes \gamma^{5} \sin \theta+\sigma^{2} \otimes \mathbf{1} \cos \theta\right) \frac{1}{a}\left[\partial_{\theta}+\frac{1}{2} i \omega \sigma^{0} \otimes \gamma^{5}-i g_{1} A_{1 \theta}\right] \psi_{\mu},(40
\end{aligned}
$$

with

$$
\omega=1-a^{\prime}, \quad g_{1} A_{1 \theta}=-n_{1}\left(\frac{1}{f_{1}}-1\right) .
$$

Using the chirality condition $\sigma^{3} \otimes \mathbf{1} \psi_{\mu}=\psi_{\mu}$ and $\gamma^{\alpha \beta \gamma} \gamma_{\beta}=-2 \gamma^{\alpha \gamma}$, we can simplify the above equation as

$$
\begin{aligned}
W^{-1} \sigma^{1} \otimes \gamma^{\alpha \beta \gamma} \delta_{\gamma}^{\mu} \partial_{\beta} \psi_{\mu}= & \sigma^{1} \otimes \gamma^{\alpha \gamma} \delta_{\gamma}^{\mu}\left[\left(\gamma^{5} \cos \theta+i \sin \theta\right)\left(\partial_{\rho}+\frac{W^{\prime}}{W}\right)\right. \\
& \left.+\left(-\gamma^{5} \sin \theta+i \cos \theta\right) \frac{1}{a}\left(\partial_{\theta}+\frac{1}{2} i \omega \gamma^{5}-i g_{1} A_{1 \theta}\right)\right] \psi_{\mu}
\end{aligned}
$$

After imposing the gauge fixing conditions, $\Gamma^{\mu} \psi_{\mu}=0$ and $\partial^{\mu} \psi_{\mu}=0$, as well as using the identity $\gamma^{\alpha \beta \gamma}=\gamma^{\alpha} \gamma^{\beta} \gamma^{\gamma}-\eta^{\alpha \beta} \gamma^{\gamma}-\eta^{\beta \gamma} \gamma^{\alpha}+\eta^{\alpha \gamma} \gamma^{\beta}$, the gravitino equation becomes

$$
\begin{aligned}
W^{-1} \sigma^{1} \otimes \gamma^{\beta} \partial_{\beta} \psi_{\mu}= & -\sigma^{1} \otimes\left[\left(\gamma^{5} \cos \theta+i \sin \theta\right)\left(\partial_{\rho}+\frac{W^{\prime}}{W}\right)\right. \\
& \left.+\left(-\gamma^{5} \sin \theta+i \cos \theta\right) \frac{1}{a}\left(\partial_{\theta}+\frac{1}{2} i \omega \gamma^{5}-i g_{1} A_{1 \theta}\right)\right] \psi_{\mu} .
\end{aligned}
$$

Finally, decomposing the $6 \mathrm{D}$ Weyl spinor $\tilde{\psi}_{\mu}$ to left and right components as $\tilde{\psi}_{\mu}=$ $\left(\tilde{\psi}_{\mu L}, \tilde{\psi}_{\mu R}\right)^{T}$, satisfying $\gamma^{5}\left(\tilde{\psi}_{\mu L}, 0\right)^{T}=+\left(\tilde{\psi}_{\mu L}, 0\right)^{T}$ and $\gamma^{5}\left(0, \tilde{\psi}_{\mu R}\right)^{T}=-\left(0, \tilde{\psi}_{\mu R}\right)^{T}$, we obtain the final form of the gravitino equation as

$$
\begin{aligned}
W^{-1} \bar{\sigma}^{\beta} \partial_{\beta} \tilde{\psi}_{\mu L} & =e^{-i \theta}\left[\partial_{\rho}+\frac{W^{\prime}}{W}+\frac{1}{a}\left(-i \partial_{\theta}-\frac{1}{2} \omega-g_{1} A_{1 \theta}\right)\right] \tilde{\psi}_{\mu R}, \\
W^{-1} \sigma^{\beta} \partial_{\beta} \tilde{\psi}_{\mu R} & =e^{i \theta}\left[-\partial_{\rho}-\frac{W^{\prime}}{W}+\frac{1}{a}\left(-i \partial_{\theta}+\frac{1}{2} \omega-g_{1} A_{1 \theta}\right)\right] \tilde{\psi}_{\mu L} .
\end{aligned}
$$




\section{Solutions to the gravitino equation}

We will now solve the above equations of motion by dimensionally reducing to 4D mass eigenstates. Therefore, we make a Fourier expansion of the "4D gravitino" as

$$
\begin{aligned}
\tilde{\psi}_{\mu L} & =\sum_{m} \tilde{\psi}_{\mu L}^{(m)}(x) \varphi_{L}^{(m)}(\rho) e^{i m \theta} \\
\tilde{\psi}_{\mu R} & =\sum_{m} \tilde{\psi}_{\mu R}^{(m)}(x) \varphi_{R}^{(m)}(\rho) e^{i m \theta}
\end{aligned}
$$

Then, plugging the above Fourier expansions into eqs.(44) and (45), we get the equations for the wave functions of the gravitino ${ }^{8}$

$$
\begin{aligned}
W^{-1} \bar{\sigma}^{\beta} \partial_{\beta} \tilde{\psi}_{\mu L}^{(m-1)} \varphi_{L}^{(m-1)} & =\left[\partial_{\rho}+\frac{W^{\prime}}{W}+\frac{1}{a}\left(m-\frac{1}{2} \omega-g_{1} A_{1 \theta}\right)\right] \tilde{\psi}_{\mu R}^{(m)} \varphi_{R}^{(m)}, \\
W^{-1} \sigma^{\beta} \partial_{\beta} \tilde{\psi}_{\mu R}^{(m+1)} \varphi_{R}^{(m+1)} & =\left[-\partial_{\rho}-\frac{W^{\prime}}{W}+\frac{1}{a}\left(m+\frac{1}{2} \omega-g_{1} A_{1 \theta}\right)\right] \tilde{\psi}_{\mu L}^{(m)} \varphi_{L}^{(m)} .
\end{aligned}
$$

The KK massive modes of gravitino are satisfying

$$
\begin{aligned}
\bar{\sigma}^{\beta} \partial_{\beta} \tilde{\psi}_{\mu L}^{(m)} & =M_{m} \tilde{\psi}_{\mu R}^{(m+1)}, \\
\sigma^{\beta} \partial_{\beta} \tilde{\psi}_{\mu R}^{(m+1)} & =M_{m} \tilde{\psi}_{\mu L}^{(m)},
\end{aligned}
$$

with $M_{m}$ the KK mass of each 4D Dirac gravitino $\tilde{\psi}_{\mu}^{(m)}=\left(\tilde{\psi}_{\mu L}^{(m)}, \tilde{\psi}_{\mu R}^{(m+1)}\right)^{T}$. Then, the equations for the gravitino wave functions become

$$
\begin{aligned}
W^{-1} M_{m-1} \varphi_{L}^{(m-1)} & =\left[\partial_{\rho}+\frac{W^{\prime}}{W}+\frac{1}{a}\left(m-\frac{1}{2} \omega-g_{1} A_{1 \theta}\right)\right] \varphi_{R}^{(m)}, \\
W^{-1} M_{m} \varphi_{R}^{(m+1)} & =\left[-\partial_{\rho}-\frac{W^{\prime}}{W}+\frac{1}{a}\left(m+\frac{1}{2} \omega-g_{1} A_{1 \theta}\right)\right] \varphi_{L}^{(m)} .
\end{aligned}
$$

The normalizability condition for the gravitino is

$$
\int d \theta \int d \rho W a\left|\varphi_{L, R}^{(m)}\right|^{2}<\infty .
$$

Furthermore, there is a hermiticity condition for the gravitino, which, in analogy with [14], is given by

$$
\int d^{6} x \partial_{N}\left(\sqrt{-G} \bar{\psi}_{M} \Gamma^{M N \Lambda} \psi_{\Lambda}\right)=0
$$

In terms of the above mode decomposition, this gives

$$
\left.W^{2} a \varphi_{L}^{(m) *} \varphi_{R}^{(m+1)}\right|_{0} ^{\rho_{s}}=0
$$

\footnotetext{
${ }^{8}$ Compare to the spin- $\frac{1}{2}$ fermions [14] charged under $U(1)_{R}$, for which the warp factor dependence in the equation comes as $2 W^{\prime} / W$ instead of $W^{\prime} / W$.
} 


\subsection{Massless modes}

For massless modes, we set $\bar{\sigma}^{\beta} \partial_{\beta} \tilde{\psi}_{\mu L}^{(m)}=\sigma^{\beta} \partial_{\beta} \tilde{\psi}_{\mu R}^{(m)}=0$ in (48), (49). Then, the equations of left-handed and right-handed gravitinos are decoupled as

$$
\begin{aligned}
{\left[\partial_{\rho}+\frac{W^{\prime}}{W}+\frac{1}{a}\left(m-\frac{1}{2} \omega-g_{1} A_{1 \theta}\right)\right] \varphi_{R}^{(m)} } & =0, \\
{\left[\partial_{\rho}+\frac{W^{\prime}}{W}+\frac{1}{a}\left(-m-\frac{1}{2} \omega+g_{1} A_{1 \theta}\right)\right] \varphi_{L}^{(m)} } & =0 .
\end{aligned}
$$

We can find the explicit solution of the above equations as

$$
\begin{aligned}
\varphi_{L}^{(m)} & =\frac{1}{W} \exp \left[\int^{\rho} d \rho^{\prime} \frac{1}{a}\left(m+\frac{1}{2} \omega-g_{1} A_{1 \theta}\right)\right] \\
& =\frac{N_{m}}{W \sqrt{a}}\left(\frac{r}{r_{0}}\right)^{\frac{s}{2}} f_{0}^{\frac{1-t}{2}}
\end{aligned}
$$

with

$$
\begin{aligned}
s & =\frac{1}{\lambda}(1+2 m), \\
t & =\frac{1}{\lambda}\left(m+\frac{1}{2}-n_{1}\right)\left(1-\frac{r_{0}^{2}}{r_{1}^{2}}\right)+\frac{n_{1}}{\lambda}+1,
\end{aligned}
$$

where $N_{m}$ is the normalization constant. In the above, we have used that

$$
\int d r \frac{f_{1}}{r f_{0}} \sim \ln \left[r f_{0}^{\frac{r_{0}^{2}-r 1^{2}}{2 r_{1}^{2}}}\right], \quad \int d r \frac{1}{r f_{0}} \sim \ln \left[r f_{0}^{-1 / 2}\right] .
$$

The solution for the right-handed gravitino is given by the one for the left-handed gravitino (58) with $\left(m, n_{1}\right)$ being replaced by $\left(-m,-n_{1}\right)$.

From the normalization condition (53), we determine the normalization constant of the general solution (58) as

$$
N_{m}^{2}=\frac{1}{2 \pi r_{0}}\left(\int_{0}^{\infty} \frac{x^{s}}{\left(1+x^{2}\right)^{t}}\right)^{-1} \equiv \frac{\Gamma_{m}}{2 \pi r_{0}}
$$

with

$$
\Gamma_{m} \equiv \frac{2 \Gamma[t]}{\Gamma[(1+s) / 2] \Gamma[t-(1+s) / 2]} .
$$

In order to have finite norm, we require the following inequalities for the existence of left-handed zero mode

$$
s>-1, \quad s-2 t<-1,
$$

or in terms of our original parameters

$$
-\frac{1}{2}(1+\lambda)<m<n_{1}-\frac{1}{2}\left(1-\lambda \frac{r_{1}^{2}}{r_{0}^{2}}\right) .
$$


For the right-handed zero mode, the normalizability conditions are

$$
n_{1}+\frac{1}{2}\left(1-\lambda \frac{r_{1}^{2}}{r_{0}^{2}}\right)<m<\frac{1}{2}(1+\lambda)
$$

Let us first discuss about the simple cases with constant warp factor. In the sphere case with $U(1)_{R}$ flux only, which is the Salam-Sezgin solution, we have the relation, $g_{1} A_{1 \theta}=\frac{1}{2} \omega$. In the above general expression, we take $r_{0}=r_{1}, \lambda=1$ and $n_{1}=1$. Then, from the normalizability conditions (64) and (65), we obtain only one massless mode (for $m=0$ ) from the left-handed gravitino as

$$
\varphi_{L}^{(0)} \propto a^{-1 / 2} r^{\frac{1}{2}} f_{0}^{-1 / 2}=\text { constant. }
$$

On the other hand, in the sphere case with $E_{6}$ flux only, i.e. $A_{1 \theta}=0$, we take $r_{0}=r_{1}$, $\lambda=1$ and $n_{1}=0$. Thus, from eqs. (64) and (65), we can see that there is no normalizable massless mode for any $m$. This shows that supersymmetry is completely broken.

Now let us look at the effects of a nonzero deficit angle and/or a warp factor. In this case, it is possible to have both $U(1)_{R}$ and $E_{6}$ fluxes non-vanishing. In the unwarped case with $\lambda \neq 1$ and $r_{0}=r_{1}$, the wave functions of massless modes are

$$
\begin{aligned}
& \varphi_{R}^{(m)} \propto a^{-1 / 2} r^{\frac{1}{\lambda}\left(\frac{1}{2}-m\right)} f_{0}^{\frac{n_{1}}{2 \lambda}}, \\
& \varphi_{L}^{(m)} \propto a^{-1 / 2} r^{\frac{1}{\lambda}\left(\frac{1}{2}+m\right)} f_{0}^{-\frac{n_{1}}{2 \lambda}} .
\end{aligned}
$$

Since $\lambda=\sqrt{n_{1}^{2}+n_{6}^{2} g_{1}^{2} / g_{6}^{2}} \geq\left|n_{1}\right|$, for $n_{1}>0$, we can see that there is at least one massless mode of $\varphi_{L}^{(m)}$ from eq. (64). Thus, although supersymmetry is broken by the $E_{6}$ flux and also explicitly by the brane sources, there exist massless modes of the gravitino. For example, in the case of $n_{6}=0, n_{1}=\lambda, q=4 g_{1}$, there exist normalizable modes for the left-handed gravitino and the nontrivial SUSY variation (27) becomes

$$
\delta \tilde{\psi}_{\theta L}=\left[\partial_{\theta}-\frac{1}{2} i\left(n_{1}-1\right)\right] \tilde{\varepsilon}_{L} .
$$

Thus, $\delta \tilde{\psi}_{\theta L}=0$ would require $\tilde{\varepsilon}_{L} \propto e^{i \frac{1}{2}\left(n_{1}-1\right) \theta}$. For even $n_{1}$, the Killing spinor is not singlevalued so there is no remaining SUSY in this case. On the other hand, for odd $n_{1}$, there is an $N=1$ SUSY left in the bulk even if SUSY is broken explicitly by the deficit angle at the brane.

Furthermore, in the case with the non-constant warp factor, we also find, from the $r_{1}^{2} / r_{0}^{2}$ term in eq. (64), that the massless modes are still maintained. Therefore, as far as $U(1)_{R}$ flux is nonzero, the massless mode of gravitino obtained in the Salam-Sezgin solution remains even with a nonzero deficit angle or a warp factor. This property of the appearance of massless modes when $U(1)_{R}$ is present, holds also for for bulk spin- $\frac{1}{2}$ fermions which are charged under the $U(1)_{R}$. Then it has been shown in $[14,18]$ that massless modes of these fermions persist after flux compactification. For the non-constant warp factor, however, there is no remaining SUSY in the bulk at all as shown from the SUSY transformations in Section 3.1. 


\subsection{Massive modes}

Returning to the massive modes, we can substitute $\varphi_{R}^{(m+1)}$ from (52) to (51) and obtain a single second order differential equation for $\varphi_{L}^{(m)}$

$$
\begin{aligned}
M_{m}^{2} \varphi_{L}^{(m)}=W & {\left[\partial_{\rho}+\frac{W^{\prime}}{W}+\frac{1}{a}\left(m+1-\frac{1}{2} \omega-g_{1} A_{1 \theta}\right)\right] } \\
& \times W\left[-\partial_{\rho}-\frac{W^{\prime}}{W}+\frac{1}{a}\left(m+\frac{1}{2} \omega-g_{1} A_{1 \theta}\right)\right] \varphi_{L}^{(m)}
\end{aligned}
$$

supplemented by eq. (52) which acts like a constraint equation. From the latter equation, the hermiticity condition (55) becomes

$$
\left.W^{3} a \varphi_{L}^{(m) *}\left[-\partial_{\rho}-\frac{W^{\prime}}{W}+\left(m+\frac{1}{2} \omega-g_{1} A_{1 \theta}\right) / a\right] \varphi_{L}^{(m)}\right|_{0} ^{\rho_{s}}=0
$$

Before tackling the most general case, let us present the solutions for the massive modes in the two simple cases of the supersymmetric (SS vacuum) and the non-supersymmetric (with $E_{6}$ flux) solutions. In both cases the internal space is a sphere and there is no warping.

\subsubsection{Massive modes for the Salam-Sezgin solution}

First, for the sphere case with a $U(1)_{R}$ flux only, $g_{1} A_{1 \theta}=\frac{1}{2} \omega$ so the equation for massive modes becomes

$$
\left[\partial_{\rho}^{2}+\frac{a^{\prime}}{a} \partial_{\rho}-\frac{m^{2}}{a^{2}}+M_{m}^{2}\right] \varphi_{L}^{(m)}=0
$$

where $a=\frac{r_{0}}{2} \sin \left(\frac{2 \rho}{r_{0}}\right)$. Then, by making a change of variables as $y=\cos \left(\frac{2 \rho}{r_{0}}\right)$, the above equation can be cast into

$$
\left(1-y^{2}\right) \frac{d^{2} \varphi_{L}^{(m)}}{d y^{2}}-2 y \frac{d \varphi_{L}^{(m)}}{d y}+\left(-\frac{m^{2}}{1-y^{2}}+\frac{1}{4} r_{0}^{2} M_{m}^{2}\right) \varphi_{L}^{(m)}=0 .
$$

This is nothing but the Legendre's associated differential equation. So, we can find that the KK mass spectrum is the one for the spherical harmonics

$$
M_{m, n}^{2}=\frac{4}{r_{0}^{2}} n(n+1), \quad n=1,2, \cdots
$$

with degeneracies $|m| \leq n$. The wave functions of massive modes are given by

$$
\varphi_{L}^{(m, n)}=P_{n}^{m}(y)=\frac{\left(1-y^{2}\right)^{m / 2}}{2^{n} n !} \frac{d^{m+n}}{d y^{m+n}}\left(y^{2}-1\right)^{n} .
$$




\subsubsection{Massive modes for the non-supersymmetric sphere solution}

In the sphere case with $E_{6}$ flux only, $A_{1 \theta}=0$, so the equation for massive modes is

$$
\left[\partial_{\rho}^{2}+\frac{a^{\prime}}{a} \partial_{\rho}-\frac{\left(m+\frac{1}{2}\right)^{2}}{a^{2}}-\frac{a^{\prime 2}}{4 a^{2}}+\frac{a^{\prime \prime}}{2 a}+\frac{\left(m+\frac{1}{2}\right) a^{\prime}}{a^{2}}+M_{m}^{2}\right] \varphi_{L}^{(m)}=0,
$$

where $a=\frac{r_{0}}{2} \sin \left(\frac{2 \rho}{r_{0}}\right)$. Then, with the field redefinition

$$
\tilde{\varphi}^{(m)}=\sqrt{a} \varphi_{L}^{(m)},
$$

the above equation becomes

$$
\left(-\partial_{\rho}^{2}+V(\rho)\right) \tilde{\varphi}^{(m)}=M_{m}^{2} \tilde{\varphi}^{(m)}
$$

where

$$
r_{0}^{2} V=v_{0}+v_{1} \tan ^{2}\left(\frac{\rho}{r_{0}}\right)+v_{2} \cot ^{2}\left(\frac{\rho}{r_{0}}\right),
$$

with

$$
v_{0}=2\left(m+\frac{1}{2}\right)^{2}, \quad v_{1}=(m+1)^{2}-\frac{1}{4}, \quad v_{2}=m^{2}-\frac{1}{4} .
$$

Next, making a change of variables and doing another field redefinition [14] as

$$
\begin{aligned}
z & =\cos ^{2}\left(\frac{\rho}{r_{0}}\right), \\
\tilde{\varphi}^{(m)} & =z^{\gamma}(1-z)^{\beta} \psi^{(m)}(z),
\end{aligned}
$$

with

$$
v_{1}=4 \gamma^{2}-2 \gamma, \quad v_{2}=4 \beta^{2}-2 \beta,
$$

we obtain the final form of the massive modes equation as

$$
z(1-z) \frac{d^{2} \psi^{(m)}}{d z^{2}}+[c-(1+a+b) z] \frac{d \psi^{(m)}}{d z}-a b \psi^{(m)}=0,
$$

where

$$
\begin{aligned}
a & =\beta+\gamma+\frac{1}{2} r_{0} M_{m}, \\
b & =\beta+\gamma-\frac{1}{2} r_{0} M_{m}, \\
c & =\frac{1}{2}+2 \gamma .
\end{aligned}
$$

In order to express the parameters $a, b, c$ in terms of the the integer $m$, we should solve the quadratic equations (83) with the definitions (80). There are two solutions for $\beta$ and 
$\gamma$, but there is no physical difference between the various choices of the solutions. Thus, from now on we make the choice

$$
\beta=\frac{1}{2}\left(m+\frac{1}{2}\right), \quad \gamma=-\frac{1}{2}\left(m+\frac{1}{2}\right)
$$

From the boundary conditions for the gravitino given in the Appendix B, we can now obtain the quantized KK masses of the gravitino. For $\gamma \geq \frac{1}{4}$ and $\beta<\frac{1}{4}$, we need $c-a=-n$ or $c-b=-n$ with $n=0,1,2, \cdots$. Both cases yield the spectrum

$$
M_{m \leq-1, n}^{2}=\frac{4}{r_{0}^{2}}\left(n+\frac{1}{2}+\gamma-\beta\right)^{2}=\frac{4}{r_{0}^{2}}(n-m)^{2}, \quad n=0,1,2, \cdots .
$$

Moreover, for $\gamma<\frac{1}{4}$ and $\beta \geq \frac{1}{4}$, we need $1+a-c=-n$ or $1+b-c=-n$ for $n=0,1,2, \cdots$. Then, for both cases we obtain the KK spectrum as

$$
M_{m \geq 0, n}^{2}=\frac{4}{r_{0}^{2}}\left(n+\frac{1}{2}-\gamma+\beta\right)^{2}=\frac{4}{r_{0}^{2}}(n+m+1)^{2}, \quad n=0,1,2, \cdots .
$$

On the other hand, the other ranges of $\gamma$ and $\beta$, as they are chosen in (88), are not possible. With the introduction of the new quantum number $n$, it is obvious that the wavefunctions should be labeled as $\tilde{\varphi}^{(m, n)}$ and the $4 \mathrm{D}$ modes accordingly $\tilde{\psi}^{(m, n)}$.

Consequently, from eqs.(89) and (90), the lowest massive modes for the left-handed gravitino are double degenerate with $\mathrm{KK}$ mass, $M_{0,0}^{2}=M_{-1,0}^{2}=\frac{4}{r_{0}^{2}}=8 g_{1}^{2}$. Since one pair of left-handed and right-handed gravitinos makes up a 4D massive Dirac gravitino from eq. (50), we find that there are two 4D massive gravitinos at the lowest KK level, $\tilde{\psi}_{\mu}^{(-1,0)}=\left(\tilde{\psi}_{\mu L}^{(-1,0)}, \tilde{\psi}_{\mu R}^{(0,0)}\right)^{T}$ and $\tilde{\psi}_{\mu}^{(0,0)}=\left(\tilde{\psi}_{\mu L}^{(0,0)}, \tilde{\psi}_{\mu R}^{(1,0)}\right)^{T}$.

\subsubsection{Massive modes for the general warped solution}

In the general warped case, we can rewrite eq. (70) in an expanded form as

$$
\left(-\partial_{\rho}^{2}+h \partial_{\rho}+g_{m}\right) \varphi_{L}^{(m)}=W^{-2} M_{m}^{2} \varphi_{L}^{(m)}
$$

with

$$
\begin{aligned}
h= & -3 \frac{W^{\prime}}{W}-\frac{a^{\prime}}{a}, \\
g_{m}= & -\frac{W^{\prime \prime}}{W}-\frac{W^{\prime 2}}{W^{2}}-\frac{1}{2} \frac{a^{\prime \prime}}{a}+\frac{1}{4} \frac{a^{\prime 2}}{a^{2}}-\frac{3}{2} \frac{a^{\prime} W^{\prime}}{a W} \\
& +\frac{1}{a}\left[\left(\frac{W^{\prime}}{W}-\frac{a^{\prime}}{a}\right)\left(m+\frac{1}{2}-g_{1} A_{1 \theta}\right)-g_{1} A_{1 \theta}^{\prime}\right] \\
& +\frac{1}{a^{2}}\left[m(m+1)+\frac{1}{4}+g_{1}^{2} A_{1 \theta}^{2}-(2 m+1) g_{1} A_{1 \theta}\right] .
\end{aligned}
$$


Let us make now the field redefinition $\tilde{\varphi}^{(m)}=W \sqrt{a} \varphi_{L}^{(m)}$ and the radial coordinate redefinition $d \rho=W d u$. The above equation can then be cast in the following Schrödinger form

$$
\left(-\partial_{u}^{2}+V\right) \tilde{\varphi}^{(m)}=M_{m}^{2} \tilde{\varphi}^{(m)},
$$

with the potential given by

$$
\begin{aligned}
V= & \partial_{u}\left(\frac{W}{a}\right)\left(m+\frac{1}{2}\right)-g_{1} \partial_{u}\left(\frac{W}{a} A_{1 \theta}\right) \\
& +\frac{W^{2}}{a^{2}}\left[m(m+1)+\frac{1}{4}+g_{1}^{2} A_{1 \theta}^{2}-(2 m+1) g_{1} A_{1 \theta}\right] .
\end{aligned}
$$

Substituting the background solutions in the above equation we find that

$$
r_{0}^{2} V=v_{0}+v_{1} \tan ^{2}\left(\frac{u}{r_{0}}\right)+v_{2} \cot ^{2}\left(\frac{u}{r_{0}}\right),
$$

with

$$
\begin{aligned}
& v_{0}=-\frac{1}{\lambda}\left(m+\frac{1}{2}\right)+\frac{1}{\lambda}\left(m+\frac{1}{2}-n_{1}\right)\left[1+\frac{2}{\lambda}\left(m+\frac{1}{2}\right)\right] \frac{r_{0}^{2}}{r_{1}^{2}}, \\
& v_{1}=\frac{1}{\lambda}\left(m+\frac{1}{2}-n_{1}\right) \frac{r_{0}^{2}}{r_{1}^{2}}+\frac{1}{\lambda^{2}}\left(m+\frac{1}{2}-n_{1}\right)^{2} \frac{r_{0}^{4}}{r_{1}^{4}}, \\
& v_{2}=-\frac{1}{\lambda}\left(m+\frac{1}{2}\right)+\frac{1}{\lambda^{2}}\left(m+\frac{1}{2}\right)^{2} .
\end{aligned}
$$

Then, making similar redefinitions as in the non-supersymmetric sphere solution,

$$
\begin{aligned}
z & =\cos ^{2}\left(\frac{u}{r_{0}}\right), \\
\tilde{\varphi}^{(m)} & =z^{\gamma}(1-z)^{\beta} \psi^{(m)}(z),
\end{aligned}
$$

the parameters in the hypergeometric equation (84) are defined as

$$
\begin{aligned}
a & =\beta+\gamma+\frac{1}{2} \sqrt{\xi_{m}+r_{0}^{2} M_{m}^{2}} \\
b & =\beta+\gamma-\frac{1}{2} \sqrt{\xi_{m}+r_{0}^{2} M_{m}^{2}} \\
c & =\frac{1}{2}+2 \gamma
\end{aligned}
$$

with

$$
v_{1}=4 \gamma^{2}-2 \gamma, \quad v_{2}=4 \beta^{2}-2 \beta,
$$

From eqs. (98), (99) and (105), we can solve for $\beta$ and $\gamma$. Without loss of generality, we make the following choice of roots

$$
\beta=\frac{1}{2 \lambda}\left(m+\frac{1}{2}\right), \quad \gamma=-\frac{1}{2 \lambda}\left(m+\frac{1}{2}-n_{1}\right) \frac{r_{0}^{2}}{r_{1}^{2}} .
$$


Furthermore, unlike the non-supersymmetric sphere solution, the following quantity is non-vanishing:

$$
\begin{aligned}
\xi_{m} & =v_{1}+v_{2}-v_{0} \\
& =\frac{1}{\lambda^{2}}\left[m+\frac{1}{2}-\frac{r_{0}^{2}}{r_{1}^{2}}\left(m+\frac{1}{2}-n_{1}\right)\right]^{2} \\
& =4(\beta+\gamma)^{2} .
\end{aligned}
$$

Using the boundary conditions at $z=0$ and $z=1$ as described in the Appendix B, we find the KK spectrum depending on the parameters $\beta$ and $\gamma$ :

For $\gamma \geq \frac{1}{4}$ and $\beta<\frac{1}{4}$,

$$
\begin{aligned}
r_{0}^{2} M_{m, n}^{2} & =4\left(n+\frac{1}{2}+\gamma-\beta\right)^{2}-\xi_{m} \\
& =4\left(n+\frac{1}{2}+2 \gamma\right)\left(n+\frac{1}{2}-2 \beta\right)
\end{aligned}
$$

For $\gamma \geq \frac{1}{4}$ and $\beta \geq \frac{1}{4}$

$$
\begin{aligned}
r_{0}^{2} M_{m, n}^{2} & =4(n+\beta+\gamma)^{2}-\xi_{m} \\
& =4 n(n+2 \beta+2 \gamma) .
\end{aligned}
$$

For $\gamma<\frac{1}{4}$ and $\beta<\frac{1}{4}$,

$$
\begin{aligned}
r_{0}^{2} M_{m, n}^{2} & =4(n+1-\beta-\gamma)^{2}-\xi_{m} \\
& =4(n+1)(n+1-2 \beta-2 \gamma)
\end{aligned}
$$

For $\gamma<\frac{1}{4}$ and $\beta \geq \frac{1}{4}$

$$
\begin{aligned}
r_{0}^{2} M_{m, n}^{2} & =4\left(n+\frac{1}{2}-\gamma+\beta\right)^{2}-\xi_{m} \\
& =4\left(n+\frac{1}{2}+2 \beta\right)\left(n+\frac{1}{2}-2 \gamma\right) .
\end{aligned}
$$

In all the above case, we take $n=0,1,2, \cdots$. We note that even with a nonzero $\xi_{m}$, the KK mass squared is always positive. Finally, it is understood that with the introduction of the quantum number $n$, the wavefunctions are labeled as $\tilde{\varphi}^{(m, n)}$ and the $4 \mathrm{D}$ modes accordingly $\widetilde{\psi}^{(m, n)}$.

\section{The brane-induced gravitino mass term}

In the previous section, we have seen that even when SUSY is broken explicitly by the presence of brane tensions, there remains at least one massless mode of the gravitino in the case with $U(1)_{R}$ flux. One way to generate mass for this lowest gravitino mode, is to include a brane-induced gravitino mass term. 
It has been known that a brane mass term for a bulk field on a codimension-two brane gives rise to the divergence of the propagator of the bulk field even at tree level [25] (see also [26] for a torus orbifold case). This classical divergence has to do with the assumption of an infinitely thin brane. This should have been expected, since it is known that it is not possible to accommodate normal matter (other than the brane tension) on a codimensiontwo brane in Einstein gravity [20]. Therefore, it is imperative to regularize the brane by introducing some thickness [21].

In this section, we compute the mass generation for the gravitino zero mode, when the brane is regularized according with the approach suggested in [22]. In this regularization, the conical singularity is cut out and replaced by a spherical cap and a ring-like brane is situated at the boundary of the spherical cap. Thus, the regularized brane has four spatial dimensions one of which is compactified on a circle. This procedure has been extended to the warped solution of 6D Salam-Sezgin supergravity in [23].

Let us now discuss the effect of a brane-induced gravitino mass term. If the ring is located at $\rho=\delta$ (instead of the codimension-two brane original position $\rho=0$ ), then the induced metric on the ring brane is

$$
d s_{5}^{2}=W^{2}(\delta) \eta_{\mu \nu} d x^{\mu} d x^{\nu}+a^{2}(\delta) d \theta^{2} \equiv h_{\hat{\mu} \hat{\nu}} d x^{\hat{\mu}} d x^{\hat{\nu}}
$$

with $\hat{\mu}, \hat{\nu}=0,1,2,3,5$. The left-handed gravitino always has a massless mode for a nonzero monopole number of the $U(1)_{R}$ and in general more than one, labeled by the winding number $m$. Let us consider a Majorana mass term for the left-handed gravitino only on the regularized brane as

$$
\mathcal{L}_{\text {brane }}=-\lambda_{0} \bar{\xi}_{\hat{\mu}} \gamma^{\hat{\mu} \hat{\nu}} \xi_{\hat{\nu}} \delta(\rho-\delta),
$$

where $\lambda_{0}$ is a dimensionless parameter and $\xi_{\hat{\mu}}$ is the 5D Majorana gravitino that is composed of the bulk left-handed gravitino $\tilde{\psi}_{\hat{\mu} L}$ as $\xi_{\hat{\mu}} \equiv\left(\tilde{\psi}_{\hat{\mu} L}, \overline{\tilde{\psi}}_{\hat{\mu} L}\right)^{T}$. Since, we have considered $\tilde{\psi}_{\hat{\mu} L}=\sum_{m} \varphi_{L}^{(m)}(\rho) \tilde{\psi}_{\hat{\mu} L}^{(m)} e^{i m \theta} \equiv \sum_{m} \chi_{\hat{\mu} L}^{(m)}$, we can rewrite the gravitino mass term in terms of the $4 \mathrm{D}$ Weyl spinors $\chi_{\hat{\mu} L}^{(m)}$ as

$$
\mathcal{L}_{\text {brane }}=-\lambda_{0}\left[\sum_{m, m^{\prime}}\left(\chi_{\mu L}^{(m)} \sigma^{[\mu} \bar{\sigma}^{\nu]} \chi_{\nu L}^{\left(m^{\prime}\right)}+\bar{\chi}_{\mu L}^{(m)} \bar{\sigma}^{[\mu} \sigma^{\nu]} \bar{\chi}_{\nu L}^{\left(m^{\prime}\right)}\right)+\cdots\right] \delta(\rho-\delta),
$$

where the ellipsis contains the mixing between $\chi_{\mu L}^{(m)}$ and $\chi_{\theta L}^{(m)}$ which can be absorbed by the $4 \mathrm{D}$ gravitino as in the bulk Lagrangian.

Ignoring the mixing between KK modes, we focus on the mass term for the massless mode. We plug the wave function of the massless mode of the left-handed gravitino (58) into eq. (114) and integrate the angle on the ring brane. Then, we can see that only the massless mode with a zero winding number gets a nonzero mass. If in the $r$ coordinate the location of the ring brane corresponding to $\rho=\delta$ is $r=1 / \Delta_{0}$, we obtain the $4 \mathrm{D}$ effective gravitino mass as

$$
\mathcal{L}_{\text {eff }}=-m_{3 / 2} \bar{\Psi}_{\mu} \gamma^{\mu \nu} \Psi_{\nu}
$$


with $\Psi_{\mu}=\left(\tilde{\psi}_{\mu L}^{(0)}, \overline{\tilde{\psi}}_{\mu L}^{(0)}\right)^{T}$ and

$$
m_{3 / 2}=\frac{\lambda_{0} \Gamma_{0}}{r_{0}} \frac{\left(r_{0} \Delta_{0}\right)^{-s}}{\left(1+1 /\left(r_{0} \Delta_{0}\right)^{2}\right)^{t-1}}
$$

For $r_{0} \Delta_{0} \gg 1$, the gravitino mass becomes

$$
m_{3 / 2} \approx \frac{\lambda_{0} \Gamma_{0}}{r_{0}}\left(r_{0} \Delta_{0}\right)^{-s}
$$

Since $s=\frac{1}{\lambda}>0$ for $m=0$, compatible with normalizability condition $s>-1$, the gravitino mass is suppressed by the delocalization of the zero mode at the regularized brane at $r=1 / \Delta_{0}$, compared to the compactification scale $1 / r_{0}$.

Similarly, for the gravitino mass term with $\lambda_{\infty}$ at the ring brane located at $r=r_{0}^{2} \Delta_{\infty}$, we obtain the $4 \mathrm{D}$ effective gravitino mass as

$$
m_{3 / 2}=\frac{\lambda_{\infty} \Gamma_{0}}{r_{0}} \frac{\left(r_{0} \Delta_{\infty}\right)^{s}}{\left(1+\left(r_{0} \Delta_{\infty}\right)^{2}\right)^{t-1}} .
$$

Thus, for $r_{0} \Delta_{\infty} \gg 1$, the gravitino mass becomes

$$
m_{3 / 2} \approx \frac{\lambda_{\infty} \Gamma_{0}}{r_{0}}\left(r_{0} \Delta_{\infty}\right)^{s-2 t+2}
$$

If we take $s-2 t+2=\frac{1}{\lambda} \frac{r_{0}^{2}}{r_{1}^{2}}\left(1-2 n_{1}\right)<0$ or $n_{1}>\frac{1}{2}$, compatible with the normalizability condition $s-2 t<-1$, we can also get a suppression of the gravitino mass due to the delocalization of the zero mode at the regularized brane at $r=r_{0}^{2} \Delta_{\infty}$. Therefore, for the zero-mode gravitino with the zero winding number, we find that gravitino mass terms localized at both regularized branes give rise to a suppressed effective gravitino mass.

For both of the above cases, if we consider the zero thickness limit of $\Delta_{0, \infty} \rightarrow \infty$, the effective gravitino mass vanishes. Therefore, we see that the nonzero gravitino mass crucially depends on the regularization of the brane. In other words, a nonzero gravitino mass depends on the mechanism to stabilize the brane thickness.

Finally, let us comment on the possibility of giving a mass to the massless mode of the gravitino by some bulk operator. A plausible case would be from the quartic fermion terms provided in [27], if gaugino condensation is realized. For instance, since $E_{7}$ gauginos are charged under $U(1)_{R}$, they will have massless modes at the compactification scale which can serve for condensation and subsequent gravitino mass generation in the 4D effective theory. Then, one should be careful with the possible backreaction to the background solution of the additional terms (see [28]) in the scalar potential that will arise after condensation, although as noted in [27] this will be absent for constant dilaton and gravitino wavefunctions. 


\section{Conclusions}

In the present paper, we discussed the spectrum of the gravitino of the six-dimensional gauged supergravity model with gauge group $E_{7} \times E_{6} \times U(1)_{R}$, where a gauge flux is turned on in the $U(1) \subset E_{6}$ and the $U(1)_{R}$ directions. We studied in detail the spectrum in the general warped background where codimension-two branes were supporting the necessary conical singularities.

An interesting result of the paper is that there exist zero modes in the gravitino spectrum even in the case where supersymmetry is explicitly broken by the presence of the non-supersymmetric branes. This seems to be a special property whenever magnetic flux lies in the $U(1)_{R}$ direction. In order to give mass to these massless modes, the explicit supersymmetry breaking by the brane tensions is not enough, and further bulk or brane operators should be considered.

We have considered for simplicity, a brane operator for the gravitino, namely a brane Majorana mass. Regularizing the brane as in $[22,23]$, we have calculated the mass that is generated for the zero mode of the gravitino. The resulting effective gravitino mass depends on the localization of the wavefunction of the zero mode and when the winding number is zero, the gravitino mass can be parametrically smaller than the compactification scale. This suppression, however, is power-like and if the compactification scale is of the order of the GUT breaking scale, we would need a mechanism to explain the smallness $\lambda_{0}$ and thus of the SUSY breaking scale.

The above property for the massless gravitino and its mass suppression with extra operators, should also hold for the other fermionic states of the spectrum which we did not consider in the present paper. In particular the gauginos which correspond to the directions of isometry of the internal space should have the same feature. This procedure offers an alternative way to obtain light (in comparison with the scale of compactification) fermions in models with extra dimensions.

\section{Acknowledgments}

H.M.L. is supported by the DOE Contracts DOE-ER-40682-143 and DEAC02-6CH03000.

\section{Appendix A: Notations and conventions}

We use the metric signature $(-,+,+,+,+,+)$ for the $6 \mathrm{D}$ metric. The index conventions are the following: (1) for the Einstein indices we use $M, N, \cdots=0, \cdots, 5,6$ for the $6 \mathrm{D}$ indices, $\mu, \nu, \cdots,=0, \cdots, 3$ for the $4 \mathrm{D}$ indices and $m, n, \cdots=5,6$ for the internal $2 \mathrm{D}$ indices, (2) for the Lorentz indices we use $A, B, \cdots=0, \cdots, 5,6$ for the $6 \mathrm{D}$ indices, $\alpha, \beta, \cdots=0, \cdots, 3$ for the $4 \mathrm{D}$ indices and $a, b, \cdots=5,6$ for the internal $2 \mathrm{D}$ indices. 


\section{A-1. Gamma matrices}

We take the gamma matrices in the locally flat coordinates [8], satisfying $\left\{\Gamma_{A}, \Gamma_{B}\right\}=2 \eta_{A B}$, to be

$$
\Gamma_{\alpha}=\sigma^{1} \otimes \gamma_{\alpha}, \quad \Gamma_{5}=\sigma^{1} \otimes \gamma_{5}, \quad \Gamma_{6}=\sigma^{2} \otimes \mathbf{1},
$$

where $\gamma^{\prime}$ s are the 4D gamma matrices with $\gamma_{5}^{2}=1$ and $\sigma$ 's are the Pauli matrices with $\left[\sigma^{i}, \sigma^{j}\right]=2 i \epsilon_{i j k} \sigma^{k}$, with $i, j, k=1,2,3$,

$$
\sigma^{1}=\left(\begin{array}{ll}
0 & 1 \\
1 & 0
\end{array}\right), \quad \sigma^{2}=\left(\begin{array}{rr}
0 & -i \\
i & 0
\end{array}\right), \quad \sigma^{3}=\left(\begin{array}{rr}
1 & 0 \\
0 & -1
\end{array}\right)
$$

The curved gamma matrices on the other hand are given in terms of the ones in the locally flat coordinates as $\Gamma^{M}=e_{A}^{M} \Gamma^{A}$ where $e_{A}^{M}$ is the $6 \mathrm{D}$ vielbein. In addition, the $6 \mathrm{D}$ chirality operator is given by

$$
\Gamma_{7}=\Gamma_{0} \Gamma_{1} \cdots \Gamma_{6}=\sigma^{3} \otimes \mathbf{1}
$$

The convention for $4 \mathrm{D}$ gamma matrices is that

$$
\gamma^{\alpha}=\left(\begin{array}{ll}
0 & \sigma^{\alpha} \\
\bar{\sigma}^{\alpha} & 0
\end{array}\right), \quad \gamma^{5}=\left(\begin{array}{rr}
\mathbf{1} & 0 \\
0 & -\mathbf{1}
\end{array}\right),
$$

with $\sigma^{\alpha}=\left(\mathbf{1}, \sigma^{i}\right)$ and $\bar{\sigma}^{\alpha}=\left(-\mathbf{1}, \sigma^{i}\right)$. The chirality projection operators are defined as $P_{L}=\left(1+\gamma^{5}\right) / 2$ and $P_{R}=\left(1-\gamma^{5}\right) / 2$.

Finally, some useful quantities which we use in the text are the following

$$
\Gamma^{\alpha 5}=\mathbf{1} \otimes \gamma^{\alpha} \gamma^{5}, \quad \Gamma^{\alpha 6}=i \sigma^{3} \otimes \gamma^{\alpha}, \quad \Gamma^{56}=i \sigma^{3} \otimes \gamma^{5}
$$

\section{A-2. Spin connection}

For the general warped solution written in the Gaussian normal coordinate

$$
d s^{2}=W^{2} \eta_{\mu \nu} d x^{\mu} d x^{\nu}+d \rho^{2}+a^{2} d \theta^{2},
$$

the nonzero vielbein components are given by

$$
\begin{aligned}
e_{\mu}^{\alpha} & =W \delta_{\mu}^{\alpha}, \\
e_{m}^{a} & =\left(\begin{array}{ll}
\cos \theta & -\sin \theta \\
\sin \theta & \cos \theta
\end{array}\right)\left(\begin{array}{ll}
1 & 0 \\
0 & a
\end{array}\right) .
\end{aligned}
$$

Therefore, the nonzero components of the spin connection are

$$
\begin{aligned}
\omega^{\alpha}{ }_{5} & =\cos \theta W^{\prime} \delta_{\mu}^{\alpha} d x^{\mu}, \\
\omega^{\alpha}{ }_{6} & =\sin \theta W^{\prime} \delta_{\mu}^{\alpha} d x^{\mu}, \\
\omega^{5}{ }_{6} & =\left(1-a^{\prime}\right) d \theta \equiv \omega d \theta,
\end{aligned}
$$


where prime denotes the derivative with respect to $\rho$.

\section{Appendix B: Boundary conditions for the gravitino}

The general solution to the hypergeometric differential equation $(84)$ is $\psi(z)$ with $\tilde{\varphi}=$ $z^{\gamma}(1-z)^{\beta} \psi(z)$ is (we have suppressed the index $m$ in all wavefunctions):

For $c \neq 1$,

$$
\tilde{\varphi}=c_{1} \varphi_{1}+c_{2} \varphi_{2}
$$

and for $c=1$,

$$
\tilde{\varphi}=c_{1} \varphi_{1}+c_{2} \varphi_{1} \int^{z(\rho)} \frac{d \rho}{\varphi_{1}^{2}(\rho)} .
$$

The $c_{1}, c_{2}$ are integration constants and

$$
\begin{aligned}
& \varphi_{1}=z^{\gamma}(1-z)^{\beta} F(a, b, c, z), \\
& \varphi_{2}=z^{\gamma}(1-z)^{\beta} z^{1-c} F(a+1-c, b+1-c, 2-c, z) .
\end{aligned}
$$

Here we note that $F(a, b, c, z)$ is the hypergeometric function which has the properties:

$$
F(a, b, c, z) \rightarrow 1 \text { for } z \rightarrow 0
$$

and

$$
\begin{aligned}
F(a, b, c, z)=\quad C_{1} F(a, b, a+b-c+1,1-z) \\
\quad+C_{2}(1-z)^{c-a-b} F(c-a, c-b, c-a-b+1,1-z),
\end{aligned}
$$

with

$$
C_{1}=\frac{\Gamma(c) \Gamma(c-a-b)}{\Gamma(c-a) \Gamma(c-b)}, \quad C_{2}=\frac{\Gamma(c) \Gamma(-c+a+b)}{\Gamma(a) \Gamma(b)} .
$$

Now we consider the boundary conditions for the wave functions at $z=0$ and $z=1$. First, for $c \neq 1$, as $z \rightarrow 0$, the wave function goes like

$$
\tilde{\varphi} \rightarrow c_{1} z^{\gamma}+c_{2} z^{\frac{1}{2}-\gamma} .
$$

So, the normalizability condition gives, $c_{1}=0$ for $\gamma \leq-\frac{1}{4}$ while $c_{2}=0$ for $\gamma \geq \frac{3}{4}$. On the other hand, the hermiticity condition gives, $c_{1}=0$ for $\gamma<\frac{1}{4}$ while $c_{2}=0$ for $\gamma>\frac{1}{4}$. We can also show that $c_{2}=0$ for $c=1$ or $\gamma=\frac{1}{4}$.

Then, for $\gamma \geq \frac{1}{4}$, where $c_{2}=0$, the wave function at $z \rightarrow 1$ behaves as

$$
\tilde{\varphi} \rightarrow C_{1}(1-z)^{\beta}+C_{2}(1-z)^{\frac{1}{2}-\beta} .
$$

Similarly, the normalizability condition gives, $C_{1}=0$ for $\beta \leq-\frac{1}{4}$ while $C_{2}=0$ for $\beta \geq \frac{3}{4}$. On the other hand, the hermiticity condition gives, $C_{1}=0$ for $\beta<\frac{1}{4}$ while $C_{2}=0$ for $\beta \geq \frac{1}{4}$. Therefore, for $\gamma \geq \frac{1}{4}$ and $\beta<\frac{1}{4}, C_{1}=0$ gives $\Gamma(c-a)=\infty$ or $\Gamma(c-b)=\infty$, i.e. 
$c-a=-n$ or $c-b=-n$ for $n=0,1,2, \cdots$. On the other hand, for $\gamma \geq \frac{1}{4}$ and $\beta \geq \frac{1}{4}$, $C_{2}=0$ requires $\Gamma(a)=\infty$ or $\Gamma(b)=\infty$, i.e. $a=-n$ or $b=-n$ for $n=0,1,2, \cdots$.

Finally, for $\gamma<\frac{1}{4}$, where $c_{1}=0$, the wave function at $z \rightarrow 1$ goes like

$$
\tilde{\varphi} \rightarrow C_{1}^{\prime}(1-z)^{\beta}+C_{2}^{\prime}(1-z)^{\frac{1}{2}-\beta}
$$

where $C_{1}^{\prime}, C_{2}^{\prime}$ are the ones obtained from $C_{1}, C_{2}$ with $a \rightarrow a+1-c, b \rightarrow b+1-c$ and $c \rightarrow 2-c$. So, again the hermiticity condition provides the strongest constraint and for $\beta<\frac{1}{4}, C_{1}^{\prime}=0$ requires $\Gamma(1-a)=\infty$ or $\Gamma(1-b)=\infty$, i.e. $1-a=-n$ or $1-b=-n$ for $n=0,1,2, \cdots$. For $\beta \geq \frac{1}{4}, C_{2}^{\prime}=0$ would require $\Gamma(1+a-c)=\infty$ or $\Gamma(1+b-c)=\infty$, i.e. $1+a-c=-n$ or $1+b-c=-n$ for $n=0,1,2, \cdots$.

\section{References}

[1] I. Antoniadis, N. Arkani-Hamed, S. Dimopoulos and G. R. Dvali, Phys. Lett. B 436 (1998) 257 [arXiv:hep-ph/9804398]; N. Arkani-Hamed, S. Dimopoulos and G. R. Dvali, Phys. Rev. D 59 (1999) 086004 [arXiv:hep-ph/9807344].

[2] L. Randall and R. Sundrum, Phys. Rev. Lett. 83 (1999) 3370 [arXiv:hep-ph/9905221].

[3] S. Weinberg, Rev. Mod. Phys. 61 (1989) 1.

[4] N. Arkani-Hamed, S. Dimopoulos, N. Kaloper and R. Sundrum, Phys. Lett. B 480 (2000) 193 [arXiv:hep-th/0001197]; S. Kachru, M. B. Schulz and E. Silverstein, Phys. Rev. D 62 (2000) 045021 [arXiv:hep-th/0001206]; J. E. Kim, B. Kyae and H. M. Lee, Phys. Rev. Lett. 86 (2001) 4223 [arXiv:hep-th/0011118].

[5] J. W. Chen, M. A. Luty and E. Ponton, JHEP 0009 (2000) 012 [arXiv:hepth/0003067].

[6] S. Randjbar-Daemi, A. Salam and J. A. Strathdee, Nucl. Phys. B 214 (1983) 491; S. M. Carroll and M. M. Guica, arXiv:hep-th/0302067; I. Navarro, JCAP 0309 (2003) 004 [arXiv:hep-th/0302129].

[7] I. Navarro, Class. Quant. Grav. 20 (2003) 3603 [arXiv:hep-th/0305014]; H. P. Nilles, A. Papazoglou and G. Tasinato, Nucl. Phys. B 677 (2004) 405 [arXiv:hep-th/0309042]; H. M. Lee, Phys. Lett. B 587 (2004) 117 [arXiv:hep-th/0309050]; J. Garriga and M. Porrati, JHEP 0408 (2004) 028 [arXiv:hep-th/0406158].

[8] H. Nishino and E. Sezgin, Phys. Lett. B 144 (1984) 187; A. Salam and E. Sezgin, Phys. Lett. B 147 (1984) 47.

[9] Y. Aghababaie, C. P. Burgess, S. L. Parameswaran and F. Quevedo, Nucl. Phys. B 680 (2004) 389 [arXiv:hep-th/0304256]. 
[10] G. W. Gibbons, R. Guven and C. N. Pope, Phys. Lett. B 595 (2004) 498 [arXiv:hepth/0307238]; Y. Aghababaie et al., JHEP 0309 (2003) 037 [arXiv:hep-th/0308064]; C. P. Burgess, F. Quevedo, G. Tasinato and I. Zavala, JHEP 0411 (2004) 069 [arXiv:hep-th/0408109].

[11] H. M. Lee and C. Ludeling, JHEP 0601 (2006) 062 [arXiv:hep-th/0510026].

[12] A. J. Tolley, C. P. Burgess, D. Hoover and Y. Aghababaie, arXiv:hep-th/0512218.

[13] H. M. Lee and A. Papazoglou, Nucl. Phys. B 747 (2006) 294 [arXiv:hep-th/0602208], Erratum-ibid. B 765 (2007) 200; C. P. Burgess, C. de Rham, D. Hoover, D. Mason and A. J. Tolley, arXiv:hep-th/0610078.

[14] S. L. Parameswaran, S. Randjbar-Daemi and A. Salvio, arXiv:hep-th/0608074.

[15] S. Kachru, R. Kallosh, A. Linde and S. P. Trivedi, Phys. Rev. D 68 (2003) 046005 [arXiv:hep-th/0301240].

[16] C. P. Burgess, arXiv:hep-th/0510123.

[17] E. Elizalde, M. Minamitsuji and W. Naylor, Phys. Rev. D 75 (2007) 064032 [arXiv:hep-th/0702098]; M. Minamitsuji, arXiv:0704.3623 [gr-qc].

[18] S. Randjbar-Daemi, A. Salam, E. Sezgin and J. A. Strathdee, Phys. Lett. B 151 (1985) 351.

[19] S. D. Avramis, A. Kehagias and S. Randjbar-Daemi, JHEP 0505 (2005) 057 [arXiv:hep-th/0504033]; S. D. Avramis and A. Kehagias, JHEP 0510 (2005) 052 [arXiv:hep-th/0508172]; R. Suzuki and Y. Tachikawa, arXiv:hep-th/0512019.

[20] J. M. Cline, J. Descheneau, M. Giovannini and J. Vinet, JHEP 0306 (2003) 048 [arXiv:hep-th/0304147].

[21] M. Kolanovic, M. Porrati and J. W. Rombouts, Phys. Rev. D 68 (2003) 064018 [arXiv:hep-th/0304148]; S. Kanno and J. Soda, JCAP 0407 (2004) 002 [arXiv:hepth/0404207]; J. Vinet and J. M. Cline, Phys. Rev. D 70 (2004) 083514 [arXiv:hepth/0406141]; J. Vinet and J. M. Cline, Phys. Rev. D 71 (2005) 064011 [arXiv:hepth/0501098]; I. Navarro and J. Santiago, JHEP 0502 (2005) 007 [arXiv:hepth/0411250]; C. de Rham and A. J. Tolley, JCAP 0602 (2006) 003 [arXiv:hepth/0511138].

[22] M. Peloso, L. Sorbo and G. Tasinato, Phys. Rev. D 73 (2006) 104025 [arXiv:hepth/0603026].

[23] E. Papantonopoulos, A. Papazoglou and V. Zamarias, JHEP 0703 (2007) 002 [arXiv:hep-th/0611311]. 
[24] M. Henneaux, Phys. Rev. D 29 (1984) 2766.

[25] W. D. Goldberger and M. B. Wise, Phys. Rev. D 65 (2002) 025011 [arXiv:hepth/0104170].

[26] E. Dudas, C. Grojean and S. K. Vempati, arXiv:hep-ph/0511001.

[27] H. Nishino and E. Sezgin, Nucl. Phys. B 278 (1986) 353.

[28] Y. Aghababaie, C. P. Burgess, S. L. Parameswaran and F. Quevedo, JHEP 0303 (2003) 032 [arXiv:hep-th/0212091]. 